\title{
QUANTIFYING THE SHORT LIFETIME WITH TCSPC-FLIM: FIRST MOMENT VERSUS FITTING METHODS
}

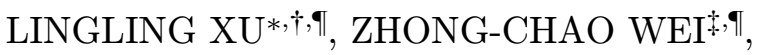 \\ SHAOQUN ZENG ${ }^{*, \dagger}$ and ZHEN-LI HUANG*, ${ }^{*, \dagger}$ \\ *Britton Chance Center for Biomedical Photonics \\ Wuhan National Laboratory for Optoelectronics-Huazhong \\ University of Science and Technology \\ Wuhan 430074, P. R. China \\ ${ }^{\dagger}$ Key Laboratory of Biomedical Photonics of Ministry of Education \\ Department of Biomedical Engineering \\ Huazhong University of Science and Technology \\ Wuhan 430074, P. R. China \\ $\$$ Laboratory of Nanophotonic Functional Materials and Devices \\ South China Normal University \\ Guangzhou, 510006, P. R. China \\ sleo@mail.hust.edu.cn
}

Received 17 June 2013

Accepted 3 July 2013

Published 31 July 2013

\begin{abstract}
Combing the time-correlated single photon counting (TCSPC) with fluorescence lifetime imaging microscopy (FLIM) provides promising opportunities in revealing important information on the microenvironment of cells and tissues, but the applications are thus far mainly limited by the accuracy and precision of the TCSPC-FLIM technique. Here we present a comprehensive investigation on the performance of two data analysis methods, the first moment $\left(M_{1}\right)$ method and the conventional least squares (Fitting) method, in quantifying fluorescence lifetime. We found that the $M_{1}$ method is more superior than the Fitting method when the lifetime is short (70 $\sim 400 \mathrm{ps})$ or the signal intensity is weak ( $<10^{3}$ photons $)$.
\end{abstract}

Keywords: Fluorescence lifetime imaging; time-correlated single photon counting; the distribution of the first moment $\left(M_{1}\right)$.

TThese authors contributed equally to this work.

This is an Open Access article published by World Scientific Publishing Company. It is distributed under the terms of the Creative Commons Attribution 3.0 (CC-BY) License. Further distribution of this work is permitted, provided the original work is properly cited. 


\section{Introduction}

The microenvironment in cells and tissues plays a crucial role in biology, including $\mathrm{pH}$, ion concentrations, oxygen saturation and other parameters. ${ }^{1,2}$ The variations of these parameters are generally very small. Thus, the methods for measuring the variations of the microenvironment parameters, should have characteristics of high spatial resolution, ${ }^{3}$ high sensitivity ${ }^{4}$ and high precision. ${ }^{5}$ There are many methods to measure the variations of microenvironment, such as magnetic resonance imaging, ${ }^{6,7}$ near-infrared spectroscopy ${ }^{8}$ and intensity imaging. ${ }^{9,10}$ However, the magnetic resonance imaging and near-infrared spectroscopy are limited by the spatial resolution, while the sensitivity and precision of the intensity imaging are low.

On the other hand, researchers combine fluorescence lifetime measurement with microscopy and build a powerful imaging technique called fluorescence lifetime imaging microscopy (FLIM), which can be used to study cell microenvironment with high spatial resolution and sensitivity. ${ }^{11}$ Obviously, the precision and accuracy of fluorescence lifetime measurements play a critical role in such studies. ${ }^{12}$

Here we focus the performance of a commonly used FLIM technique which is based on time-correlated single-photon counting (TCSPC) for lifetime measurement. ${ }^{13}$ In the TCSPC-FLIM technique, the least squares (Fitting) method is typically used in the analysis of fluorescence lifetime. With this Fitting method, the shortest fluorescence lifetime that can be measured is often limited by the instrument response function (IRF) of the detector used. ${ }^{13}$ However, for many of the commercial TCSPC-FLIM system, the IRF width is $\sim 200 \mathrm{ps},{ }^{14}$ indicating that the shortest lifetime that can be accurately measured with this system is approximate to $200 \mathrm{ps}$. Unfortunately, this is not sufficient for studying the lifetime shorter than the IRF width. On the other hand, we notice that another lifetime analysis method which relies on the distribution of the first moment $\left(M_{1}\right)$ of the photon arrival times ${ }^{15,16}$ may find promising applications in such studies: the accuracy of lifetime calculation in the $M_{1}$ method can be much shorter than the IRF width when the signal intensity is high. ${ }^{14}$ However, there is almost no systematic investigation on the capability and limitation of the $M_{1}$ method in TCSPC-FLIM. And, it is still unknown on how short can we measure the fluorescence lifetime with the $M_{1}$ method in a given TCSPC-FLIM system.
In this paper, through numerical simulation and experimental analysis, we investigated the performance of the $M_{1}$ and the Fitting methods in fluorescence lifetime analysis. We found that the $M_{1}$ method provides much better performance than the Fitting method in certain conditions.

\section{Methods}

\subsection{Principles of the fitting and $M_{1}$ methods}

In TCSPC-FLIM, laser pulses with short duration (typically less than the IRF width of a TCSPCFLIM system) excite a fluorescent sample and the consecutive fluorescence emission from the sample is recorded. The fluorescence intensity profile $F(t)$, which can be a single or multiexponential decay, is defined as ${ }^{17,18}$

$$
F(t)=\sum_{i} a_{i} \mathrm{e}^{-t / \tau_{i}}, \quad \sum_{i} a_{i}=1,
$$

where $a_{i}$ is the contribution and $\tau_{i}$ is the lifetime of the component $i$. In real experiments, the detected intensity profile $I(t)$ is the convolution product of $F(t)$, the fluorescence background and the IRF of the TCSPC-FLIM system, and thus can be described by ${ }^{14,17}$

$$
I(t)=(F(t)+\text { Background }) \otimes I R F .
$$

In most cases, the fluorescence lifetime $\tau_{i}$ can be determined using the least squares fitting method to successively minimize the difference between collected fluorescence decays and the theoretical intensity profile $I(t)$. Hereafter this treatment is called the Fitting method.

For calculating short lifetimes or tiny lifetime changes, the first moment of the photon distributions $\left(M_{1}\right)$ can be used. The $M_{1}$ in a TCSPCFLIM system is defined as ${ }^{16,19}$

$$
M_{1}=\frac{\sum N_{i} t_{i}}{N},
$$

where $t_{i}$ is the time of the time channel $i, N_{i}$ is the number of photons in time channel $i$, and $N$ is the total number of the time channels. The fluorescence lifetime $\tau$ is obtained from the $M_{1}$ difference between the fluorescence decay $\left(M_{1 \text { fluo }}\right)$ and the IRF $\left(M_{1 \mathrm{IRF}}\right)$.

$$
\tau=M_{1 \text { fluo }}-M_{1 \mathrm{IRF}} .
$$




\subsection{Accuracy and precision calculation}

The accuracy of fluorescence lifetime measurement is defined as ${ }^{17}$

$$
\text { Accuracy }=\frac{\tau_{\text {measured }}}{\tau_{\text {true }}},
$$

where $\tau_{\text {true }}$ is the true lifetime and $\tau_{\text {measured }}$ is the measured lifetime obtained by the $M_{1}$ or the Fitting methods. According to statistical laws, the lifetime is considered to be accurate when the accuracy is between 0.9 and 1.1. ${ }^{17}$

For the precision of lifetime measurement, we typically use the reduced chi-squared distribution $\left(\chi^{2}\right)$ for the Fitting method. ${ }^{14,17,20}$

$$
\chi^{2}=\frac{1}{Q-p} \sum_{k=1}^{n} \frac{\left(D_{k}-I_{k}\right)^{2}}{D_{k}},
$$

where $Q$ is the number of data points, $p$ is the number of fitting parameters, $D$ is the experimental data points and $I$ is the theoretical value. Note that the expected average value of $\chi^{2}$ is 1 .

The precision of the $M_{1}$ method can be determined by the standard deviation of the fluorescence lifetime $\left(\sigma_{\tau}\right)$, which can be calculated from the sum of the standard deviation of $M_{1 \text { fluo }}$ and $M_{1 \mathrm{IRF}}{ }^{21}$

$$
\sigma_{\tau}=\sqrt{\sigma_{M_{1 \text { fluo }}^{2}}^{2}+\sigma_{M_{1 \mathrm{IRF}}}^{2}} .
$$

\subsection{Simulation and analysis of FLIM data sets}

To evaluate the accuracy and precision of the lifetime calculation by the Fitting and the $M_{1}$ methods, a large number of data sets or photon histograms with controlled and known parameters were generated with a reported Monte Carlo approach. ${ }^{22}$ First, the parameters were set, including fluorescence lifetime, total photons, the number of time channels, total time window and fluorescence background. Then, histograms were built with these parameters according to the reported Monte Carlo approach and Eq. (1). Note that the time interval $(t)$ in Eq. (1) is determined by the total time window divided by the number of time channel, and the total time window (12.5 ns in our case) is limited by the repetition frequency of the pulse laser. Finally, the convolution was applied to the histograms generated in the previous step using
Eq. (2), which presents the fluorescence decay histograms ready for further analysis. Here the IRF was assumed to have a Gaussian shape whose full width at half maximum (FWHM) was determined experimentally (see Sec. 3.1).

Fluorescence lifetime can be calculated from the simulated fluorescence decay histograms using either the $M_{1}$ method [see Eqs. (3) and (4)] or the Fitting method. For the latter, the data set needs to be written into the ".sdt" file format that can be analyzed with a commercial software called SPCImage (version 3.2, Becker \& Hickl). Furthermore, the $\chi^{2}$ value was read from the SPCImage software to show the precision of lifetime measurement with the Fitting method. For the precision of lifetime measurement using the $M_{1}$ method, Eq. (7) was used.

All analysis were performed with Matlab (MathWorks) in a standard computer unless specifically mentioned. For each data point, a total number of 1000 simulated decays was used. And, the histograms were generated only for single exponential fluorescence decay.

\subsection{TCSPC-FLIM system}

The lifetime measurement was from a recent developed advantageous multispectral TCSPCFLIM system, where a 16-anode PMT detector is used to provide simultaneous 16 wavelength channels imaging without wavelength scanning. ${ }^{23}$ The system is based on an Olympus FV1000 confocal microscope, a frequency double pulse laser (generated by the Ti:Sapphire femtosecond laser, Mai Tai BB, Newport), ${ }^{24}$ a multi-spectral detector (PML-Spec, Becker \& Hickl) equipped with a 16channel PMT (Hamamatsu R5900U-01-L16) and a polychromator (Oriel MS 125 77400), and a TCSPC module (SPC-830, Becker \& Hickl). The detector is connected to a confocal detection port of the FV1000 scan head via a multimode fiber (Pure fused silica, $800 \mu \mathrm{m}$ core diameter). A detail description to the system can be found in one of our previous papers. ${ }^{25}$

In this paper, Rhodamine B in dilute aqueous solution was used as a calibration sample. For this RhB solution, the excitation wavelength was $435 \mathrm{~nm}$, the detection wavelength was $470-670 \mathrm{~nm}$, and the data acquisition was controlled by "single" operation mode of the SPCM software (Becker \& Hickl). The FLIM data were analyzed with the 
SPCImage software (Becker \& Hickl), Matlab (MathWorks) or Origin (Microcal).

\section{Results and Discussion}

\subsection{Determining the profile and timing stability of the IRF}

The IRF profile of a TCSPC-FLIM system is required for calculating the fluorescence lifetime of an unknown sample [see Eqs. (2) and (4)]. Therefore, we tried different approaches, including scattering, reflection ${ }^{26}$ and second harmonic generation (SHG), ${ }^{27}$ to characterize the IRF of our TCSPCFLIM system. We found that the IRF measurement using the SHG signal from Urea powder provides the best data quality. A representative IRF profile is shown in Fig. 1(a). The IRF was found to have FWHM of $191 \mathrm{ps,}$ which is consistent with the reported value in the literature $(150-200 \mathrm{ps}) .{ }^{14}$

On the other hand, it was reported that the lifetime accuracy of an TCSPC-FLIM system is limited by systematic timing errors rather than by the photon statistics. ${ }^{19}$ Therefore, after $30 \mathrm{~min}$ of warm-up, we recorded a series of 10 IRF curves within $10 \mathrm{~min}$, while for each measurement the data acquisition time was $5 \mathrm{~s}^{19}$ The total photon counts for each measurement are $\sim 8 \times 10^{5}$. The drift of the first moment ( $M_{1}$ drift) was calculated from the curves, and the results are shown in Fig. 1(b). We found that the mean $M_{1}$ drift within 10 min for 1024 time channels (2.3 ps) is larger than that for 4096 time channels (1.2 ps). These findings are consistent with the results of Backer et al. ${ }^{19}$

\subsection{Validating the quality of the simulated data}

In this paper, the fluorescence decay histograms were generated using the Monte Carlo approach reported by Spriet et al. ${ }^{14,22}$ In order to validate the data quality, we firstly generated a set of test histograms with the following parameters: fluorescence lifetime $=2 \mathrm{~ns}$, total photons $=10^{5}$, the number of time channels $=256$, total time windows $=12.5 \mathrm{~ns}$, fluorescence background $=0$. Then, noises that follow a Poisson distribution were added to the histograms, since the noise originated from photon background follows Poisson distribution. ${ }^{28}$ Finally, the simulated histograms were analyzed using the commercial software SPCImage (version 3.2, Becker \& Hickl). This process was repeated 1000 times and the mean fluorescence lifetime obtained from the software was found to be the same as we expected ( $2 \mathrm{~ns})$. This finding indicates that we can use the Monte Carlo approach to generate more simulated histograms for further studies.

\subsection{Determining the accuracy and precision of fluorescence lifetime measurement with extremely high signal intensity}

We used simulated fluorescence histograms where the lifetime varies from 0.02 to $5 \mathrm{~ns}$ to investigate the accuracy and precision of fluorescence lifetime measurement. To ensure a sufficient high signal quality, the total photons for each decay were set to be $10^{5}$. The results are shown in Fig. 2 .

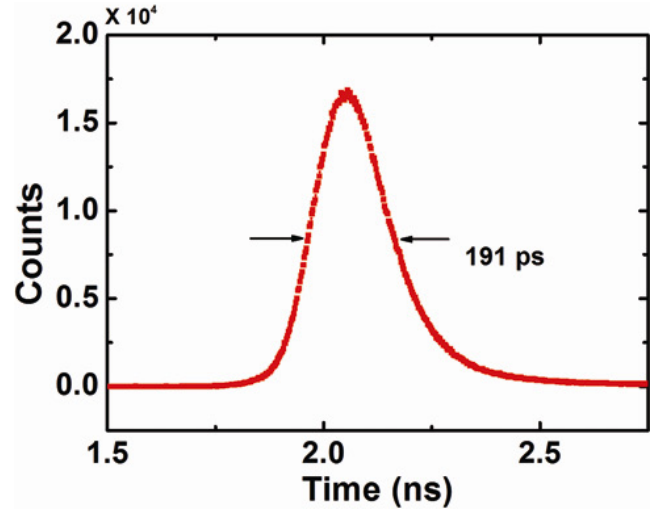

(a)

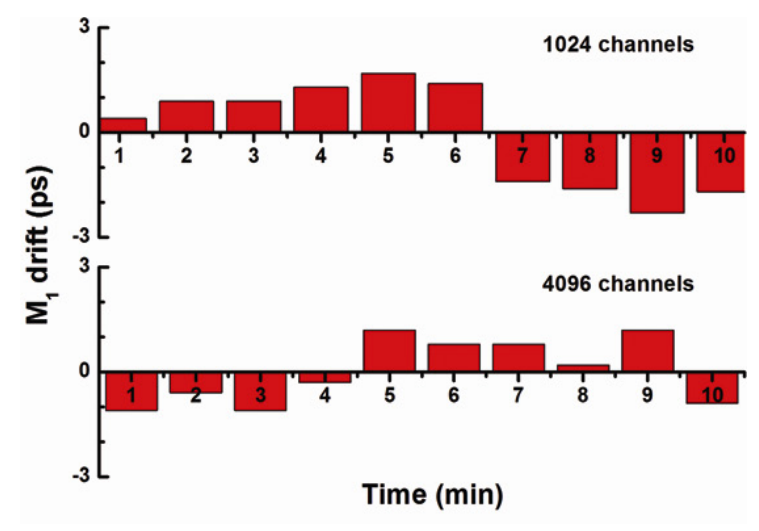

(b)

Fig. 1. (a) The IRF profile of our TCSPC-FLIM system, and (b) the $M_{1}$ drift recorded with different time channels. 


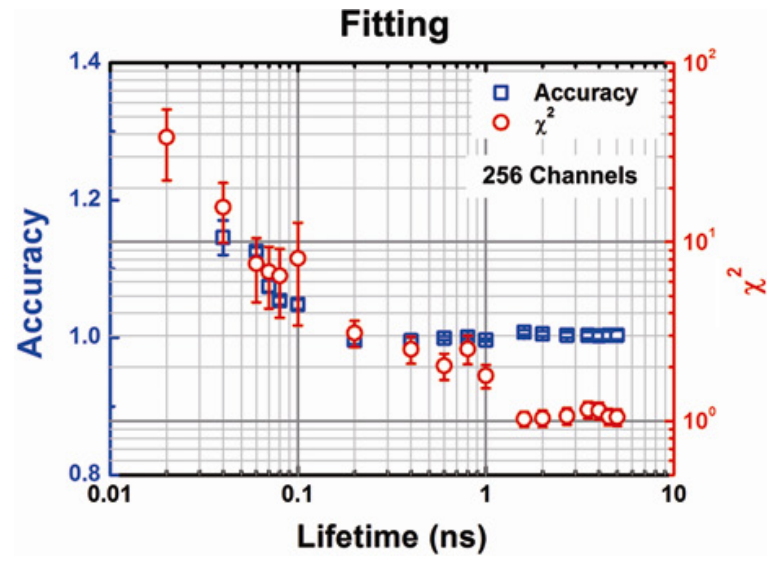

(a)

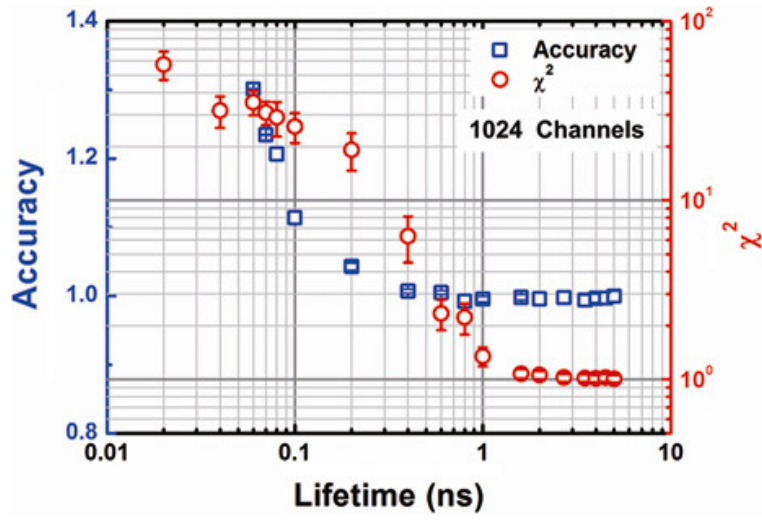

(b)

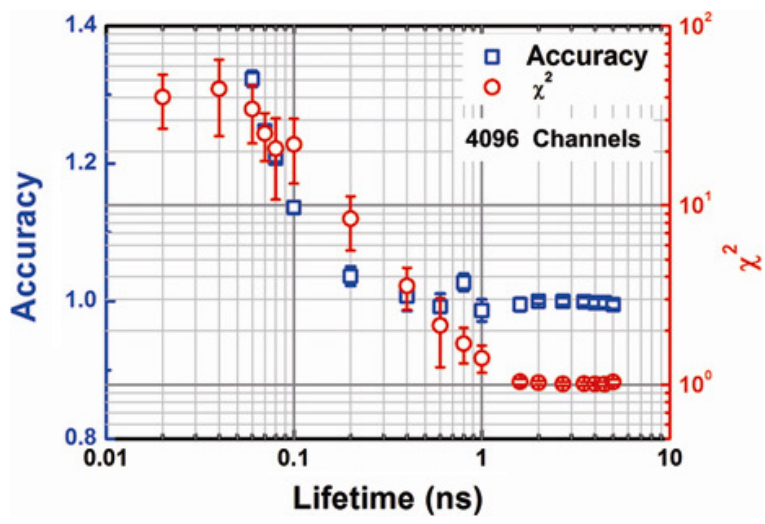

(c)

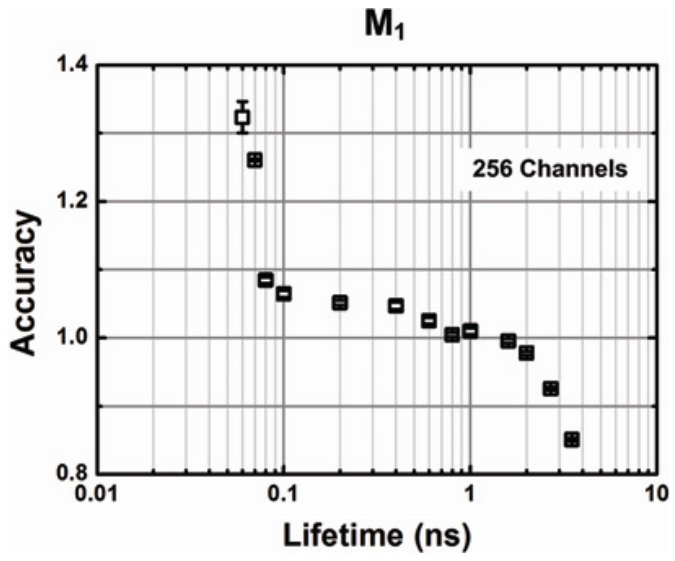

(d)

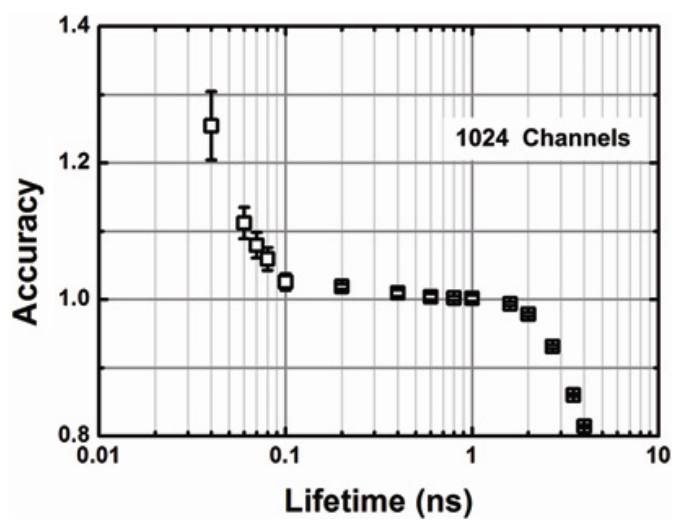

(e)

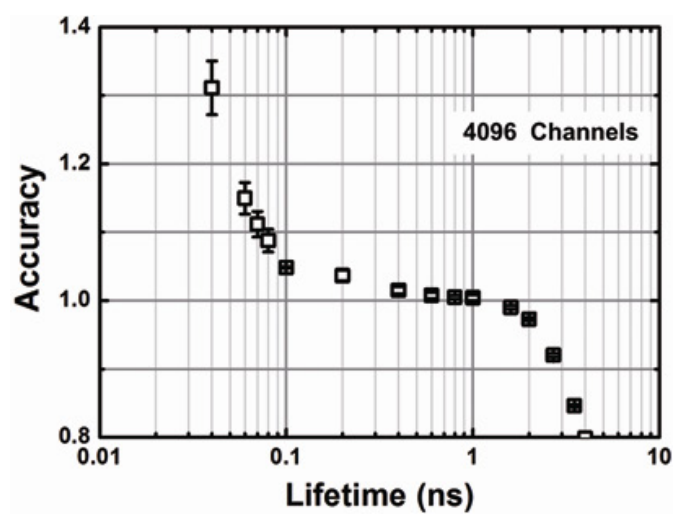

(f)

Fig. 2. The accuracy and precision of lifetime measurement using the Fitting and the $M_{1}$ methods. The signal $N=10^{5}$, and the data are from 1000 repeated measurements.

Before making any further analysis with the Fitting method, we need to identify what parameters should be used to evaluate the goodness of fit. As discussed in Sec. 2.2, the reduced chi-squared distribution $\left(\chi^{2}\right)$ is often used to evaluate the precision of the Fitting method [see Eq. (6)]. Theoretically, considering that the $N$ (equals to the number of time channels) is sufficiently large (typically larger than 100) and the $p$ is relatively small (less than 5 for single exponential decay), the 
calculated fluorescence lifetime is reliable only when the $\chi^{2}$ is smaller than 1.5 (at the $0.1 \%$ significance level). ${ }^{29}$ However, the user's handbook of the SPCImage software shows that the calculated fluorescence lifetime is still acceptable when the $\chi^{2}$ is smaller than 2.5. Note that the reasons are not explained in that handbook. Here we adopt the latter value for quantifying the goodness of fit.

From the left column in Fig. 2, we found that the accuracy of the lifetime measured from the Fitting method is sufficient when the lifetime is longer than $100 \mathrm{ps}$; however, the precision from such measurements is acceptable when the lifetime is at least $400 \mathrm{ps}$. Therefore, the shortest measurable lifetime which can be determined by the Fitting method is $\sim 400 \mathrm{ps,}$ which is about two times the IRF of the system.

The right column shows the results for the $M_{1}$ method. We found that fluorescence lifetime can be determined with sufficient accuracy and precision if the lifetime is between $70 \mathrm{ps}$ and $3 \mathrm{~ns}$. This suggests that the $M_{1}$ method is a good complimentary for the Fitting method by extending the shortest reliable lifetime measurement from 400 to $70 \mathrm{ps}$. For lifetimes shorter than $70 \mathrm{ps}$, the $M_{1}$ method is still not applicable.

\subsection{Determining the accuracy of fluorescence lifetime measurement with a fixed fluorescence lifetime}

To investigate the dependence of the accuracy of lifetime measurement on the signal intensity, we simulated a series of fluorescence decay histograms where the fluorescence lifetime was set to be $1.6 \mathrm{~ns}$. The lifetime value was chosen for the following two reasons: (1) suitable for applying both the Fitting and the $M_{1}$ methods, (2) accessible from experimental measurement (that is, Rhodamine $\mathrm{B}$ in dilute aqueous solution in this study). The results are shown in Fig. 3 which were obtained by statistical analysis of 1000 decay curves.

For the lifetime analysis using the Fitting method, we found that the accuracy depends not only on the signal intensity, but also on the number of time channels. If more time channels are used, the required minimal signal intensity should be increased significantly to keep the same accuracy and/or precision. For example, for obtaining a lifetime with a good accuracy $(1.60 \pm 0.03 \mathrm{~ns})$, a total number of $2 \times 10^{3}$ photons is sufficient under 256 time channels, while the signal should be increased to $\sim 2 \times 10^{4}$ photons when 4096 time channels are used [see Figs. 3(a) and 3(c)]. On the other hand, for the lifetime analysis using the $M_{1}$ method, we found with surprise that the accuracy depends neither on the signal intensity nor on the number of time channels, while the precision decreases with the signal intensity for all the three cases [see Figs. 3(d)-3(f), or see Sec. 3.5 for more details].

The findings above demonstrate that the $M_{1}$ method is better than the Fitting method when the signal is weak. However, we should keep in mind that the $M_{1}$ method is unfortunately sensitive to fluorescence background. Therefore, we need to perform proper background reduction before applying the $M_{1}$ method to analyze the fluorescence decay histograms.

\subsection{The dependence between signal intensity and lifetime precision}

In the previous section, the relations between lifetime precision and signal intensity were shown for different time channels separately, which is not sufficient to obtain a whole map on such relations. Here we investigate such relations with data not only from simulation, but also from experimental measurements and theoretical calculations. We found that the lifetime precision depends on both the signal intensity and the number of time channels if the Fitting method is used [see Fig. 4(a)], while for the $M_{1}$ method the lifetime precision depends mainly on the signal intensity [see Fig. 4 (b)]. These findings are consistent with those in Sec. 3.4.

Furthermore, as can be seen from Fig. 4(c), there is a good agreement between the simulated and experimental precision for Rhodamine $\mathrm{B}$ in aqueous solution, while the theoretical precision $\left(\sigma_{\tau}=\right.$ $\tau / \sqrt{N}) .{ }^{14}$ is $\sim 2$ times overestimated for different signal intensities. Nevertheless, we can still formulate the lifetime precision as a function about the total signal intensity, which would be useful for characterizing the measurement quality for further experiments. From both the simulated and experimental data, the function is:

$$
\log \left(N_{\text {count }}\right)=-1.015 \times \log \left(\sigma_{\tau}\right)+6.663,
$$

where $N_{\text {count }}$ is the total signal intensity and $\sigma_{\tau}$ is the lifetime precision obtained by the $M_{1}$ method. 


\section{Fitting}

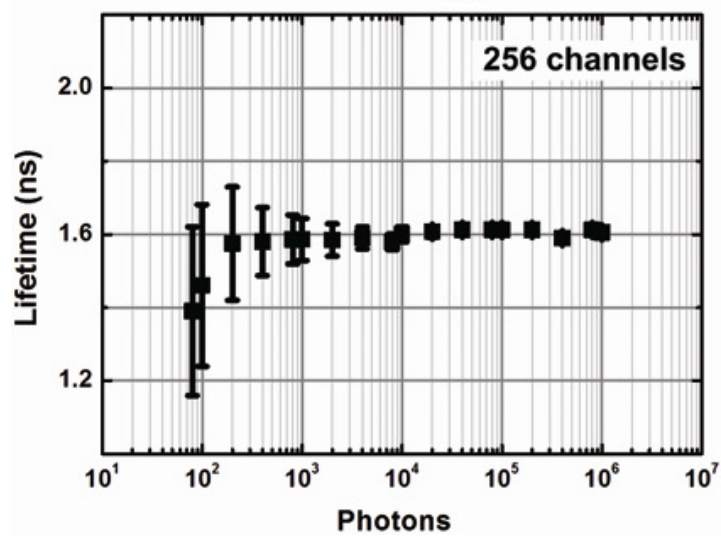

(a)

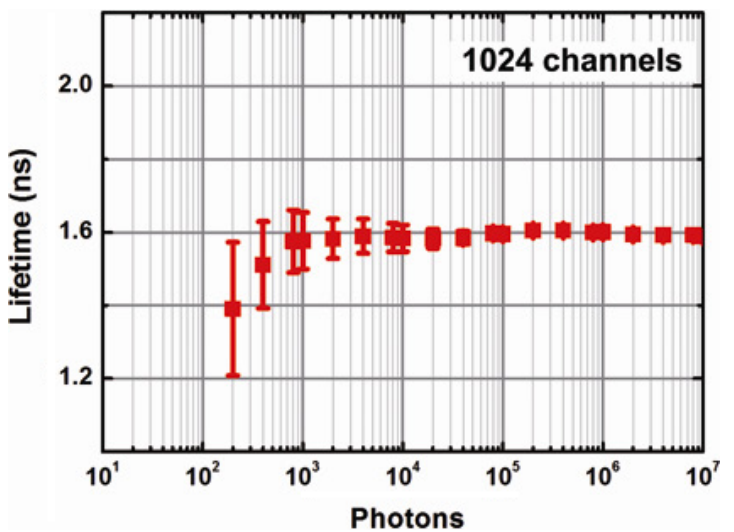

(b)

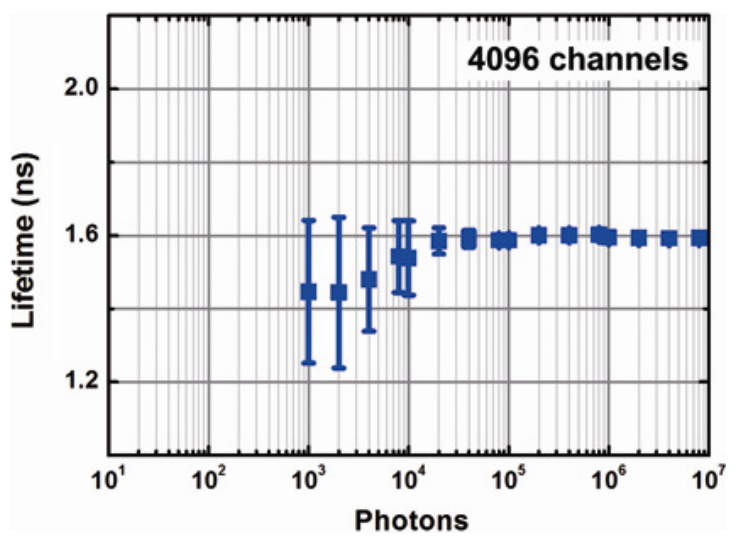

(c)

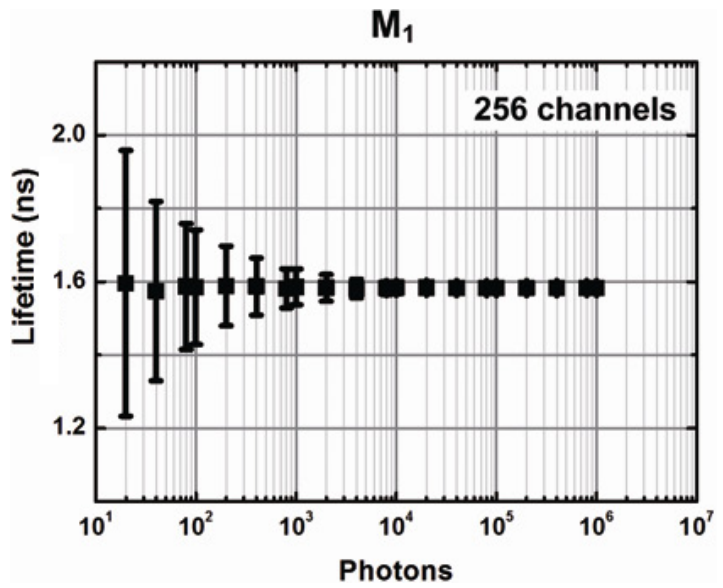

(d)

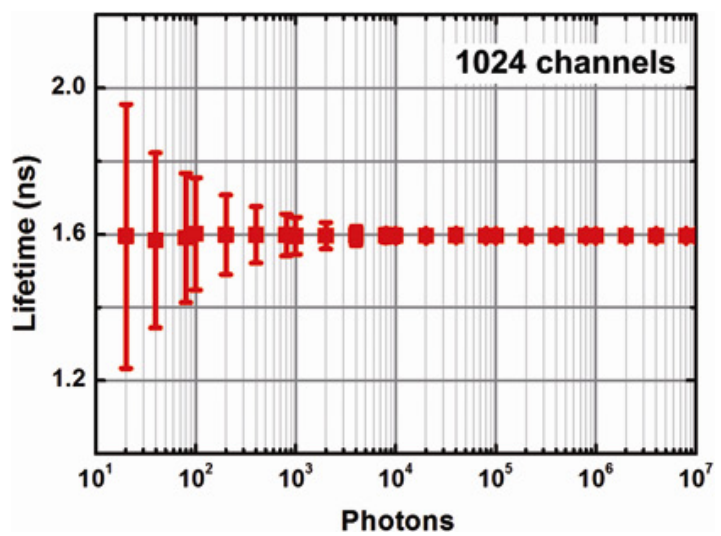

(e)

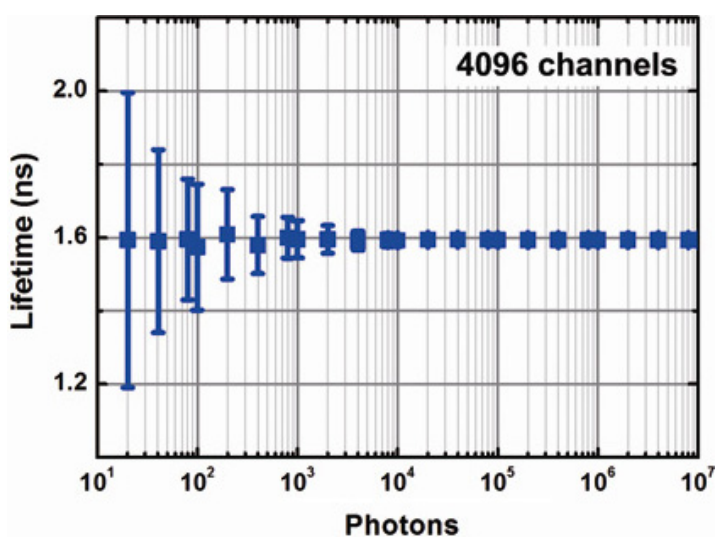

(f)

Fig. 3. Accuracy and precision of the lifetime measurement under different signal intensities and different number of time channels. Results from the Fitting and the $M_{1}$ methods are shown in the left and right column, respectively. The statistics is from 1000 repeated analysis, and the error bars represent the standard deviation of the corresponding lifetime values. 


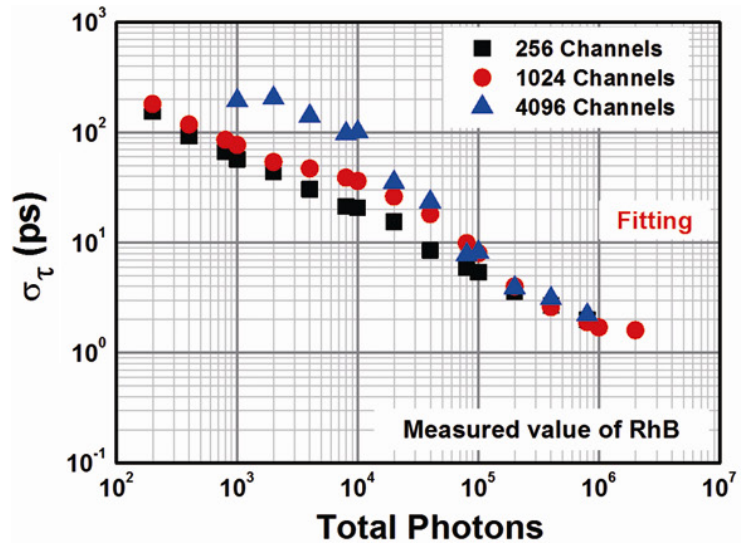

(a)

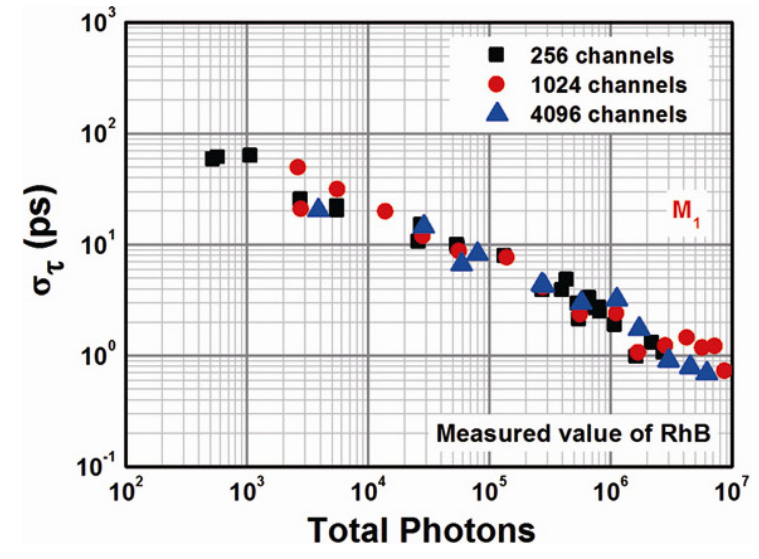

(b)

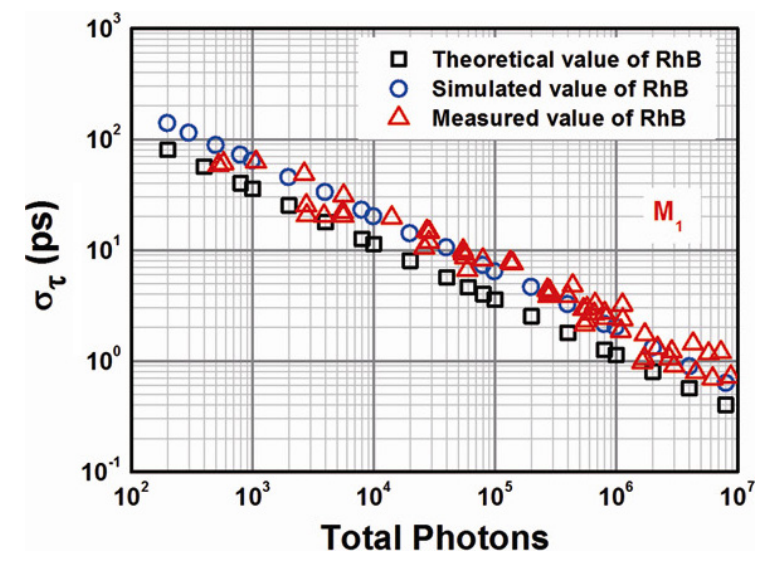

(c)

Fig. 4. The dependence between lifetime precision and signal intensity.

\section{Conclusion}

In this study, we compared the capability of the first moment $\left(M_{1}\right)$ method with the conventional least squares fitting method in quantifying fluorescence lifetime. Through simulation study, we found that the $M_{1}$ method is capable of analyzing lifetime between $70 \mathrm{ps}$ and $3 \mathrm{~ns}$ with satisfactory accuracy and precision. We also observed that, unlike the Fitting method, the performance of the $M_{1}$ method has no obvious dependence on the signal intensity and the number of time channels. Furthermore, we discovered that the $M_{1}$ method is more superior than the Fitting method if (1) the lifetime is between $70 \mathrm{ps}$ and $400 \mathrm{ps}$, or (2) the signal intensity is $<10^{3}$ photons. We believe this research will provide promising opportunities for applying the TCSPC-FLIM technique in studying the cell microenvironment with high spatial resolution, sensitivity and precision.

\section{Acknowledgment}

This work was supported by National Basic Research Program of China (Grant No. 2011CB910401), the Science Fund for Creative Research Group of China (Grant No. 61121004), the National Natural Science Foundation of China (Grant Nos. 30970691 and 61275059), the Scientific Research Foundation for the Returned Overseas Chinese Scholars, State Education Ministry of China, and the Program for New Century Excellent Talents in University of China (Grant No. NCET-10-0407). We thank Fang Long from Huazhong University of Science and Technology for technical assistance.

\section{References}

1. P. P. Hsu, D. M. Sabatini, "Cancer cell metabolism: Warburg and beyond," Cell 134, 703 (2008). 
2. A. Ostman, "The tumor microenvironment controls drug sensitivity," Nat. Med. 18, 1332-1334 (2012).

3. C. Stringari, R. A. Edwards, K. T. Pate, M. L. Waterman, P. J. Donovan, E. Gratton, "Metabolic trajectory of cellular differentiation in small intestine by phasor fluorescence lifetime microscopy of NADH," Sci. Rep. 2, srep00568 (2012).

4. R. Yasuda, C. D. Harvey, H. N. Zhong, A. Sobczyk, L. van Aelst, K. Svoboda, "Supersensitive Ras activation in dendrites and spines revealed by twophoton fluorescence lifetime imaging," Nat. Neurosci. 9, 283-291 (2006).

5. T. Nakabayashi, S. Oshita, R. Sumikawa, F. Sun, M. Kinjo, N. Ohta, "pH dependence of the fluorescence lifetime of enhanced yellow fluorescent protein in solution and cells," J. Photochem. Photobiol. A-Chemistry 235, 65-71 (2012).

6. F. A. Gallagher, M. I. Kettunen, S. E. Day, D. E. $\mathrm{Hu}$, J. H. Ardenkjaer-Larsen, R. in't Zandt, P. R. Jensen, M. Karlsson, K. Golman, M. H. Lerche, K. M. Brindle, "Magnetic resonance imaging of $\mathrm{pH}$ in vivo using hyperpolarized (13)C-labelled bicarbonate," Nature 453, 940-943 (2008).

7. D. Zhao, S. Ran, A. Constantinescu, E. W. Hahn, R. P. Mason, "Tumor oxygen dynamics: Correlation of in vivo MRI with histological findings," Neoplasia 5, 308-318 (2003).

8. M. N. Xia, V. Kodibagkar, H. L. Liu, R. P. Mason, "Tumour oxygen dynamics measured simultaneously by near-infrared spectroscopy and F-19 magnetic resonance imaging in rats," Phys. Med. Biol. 51, 45-60 (2006).

9. G.-J. Kremers, D. W. Piston, M. W. Davidson, "Fundamental principles of Förster resonance energy transfer (FRET) microscopy with fluorescent proteins," (2000-2013) Available at http://www. microscopyu.com/articles/fluorescence/fret/fretintro.html.

10. M. R. Hight, D. D. Nolting, E. T. McKinley, A. D. Lander, S. K. Wyatt, M. Gonyea, P. Zhao, H. C. Manning, "Multispectral fluorescence imaging to assess $\mathrm{pH}$ in biological specimens," J. Biomed. Opt. 16, 016007 (2011).

11. M. Y. Berezin, S. Achilefu, "Fluorescence lifetime measurements and biological imaging," Chem. Rev. 110, 2641-2684 (2010).

12. C. W. Chang, M. A. Mycek, "Precise fluorophore lifetime mapping in live-cell, multi-photon excitation microscopy," Opt. Express 18, 8688-8696 (2010).

13. Y. S. Sun, R. N. Day, A. Periasamy, "Investigating protein-protein interactions in living cells using fluorescence lifetime imaging microscopy," Nat. Protoc. 6, 1324-1340 (2011).
14. W. Becker, The bh TCSPC Handbook, 4th Edition, Becker \& Hickl GmbH, Germany (2010).

15. A. Esposito, H. C. Gerritsen, F. S. Wouters, "Fluorescence lifetime heterogeneity resolution in the frequency domain by lifetime moments analysis," Biophys. J. 89, 4286 (2005).

16. W. Becker, Advanced Time-Correlated Single Photon Counting Techniques, 1st Edition, Springer, Berlin (2005).

17. D. Trinel, A. Leray, C. Spriet, Y. Usson, L. Heliot, "Upgrading time domain FLIM using an adaptive Monte Carlo data inflation algorithm," Cytometry A 79A, 528-537 (2011).

18. V. Sharma, N. Patel, J. Shen, L. Tang, G. Alexandrakis, H. Liu, "A dual-modality optical biopsy approach for in vivo detection of prostate cancer in rat model," J. Innov. Opt. Health Sci. 4, 269-277 (2011).

19. W. Becker, A. Bergmann, "Timing stability of TCSPC experiments," in Advanced Photon Counting Techniques, W. Becker, Ed., Proc. SPIE 6372, 637209 (2006).

20. W. Becker, SPCImage 3.2 Data Analysis Software for Fluorescence Lifetime Imaging Microscopy, Becker \& Hickl GmbH, Berlin (2010).

21. Y. Won, S. Moon, W. Yang, D. Kim, W. T. Han, D. Y. Kim, "High-speed confocal fluorescence lifetime imaging microscopy (FLIM) with the analog mean delay (AMD) method," Opt. Express 19, 3396-3405 (2011).

22. C. Spriet, D. Trinel, F. Riquet, B. Vandenbunder, Y. Usson, L. Heliot, "Enhanced FRET contrast in lifetime imaging," Cytometry A 73A, 745-753 (2008).

23. W. Becker, A. Bergmann, C. Biskup, "Multispectral fluorescence lifetime imaging by TCSPC," Microsc. Res. Tech. 70, 403-409 (2007).

24. T. A. Smith, L. M. Hirvonen, C. N. Lincoln, X. Hao, "Deep-UV confocal fluorescence imaging and superresolution optical microscopy of biological samples," J. Innov. Opt. Health Sci. 5, 1250025 (2012).

25. L. Xu, L. Wang, Z. Zhang, Z.-L. Huang, "A feasible add-on upgrade on a commercial two-photon FLIM microscope for optimal FLIM-FRET imaging of CFP-YFP Pairs," J. Fluorescence 23, 543-549 (2013).

26. M. Szabelski, R. Luchowski, Z. Gryczynski, P. Kapusta, U. Ortmann, I. Gryczynski, "Evaluation of instrument response functions for lifetime imaging detectors using quenched Rose Bengal solutions," Chem. Phys. Lett. 471, 153-159 (2009).

27. C. B. Talbot, R. Patalay, I. Munro, S. Warren, F. Ratto, P. Matteini, R. Pini, H. G. Breunig, K. Konig, A. C. Chu, G. W. Stamp, M. A. A. Neil, P. M. W. French, C. Dunsby, "Application of ultrafast 
L. Xu et al.

gold luminescence to measuring the instrument response function for multispectral multiphoton fluorescence lifetime imaging," Opt. Express 19, 13848-13861 (2011).

28. F. Long, S. Q. Zeng, Z. L. Huang, "Localizationbased super-resolution microscopy with an sCMOS camera Part II: Experimental methodology for comparing sCMOS with EMCCD cameras," Opt. Express 20, 17741-17759 (2012).

29. J. R. Taylor, An Introduction to Error Analysis: The Study of Uncertainties in Physical Measurements, University Science Books, Sausalito, California (1997). 